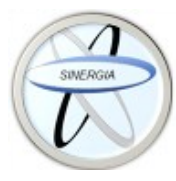

Revista Médica Sinergia

Vol. 6, Núm. 5, mayo 2021, e667

https://doi.org/10.31434/rms.v6i5.667

revistamedicasinergia@gmail.com

\title{
Incontinencia urinaria
}

\section{Urinary incontinence}

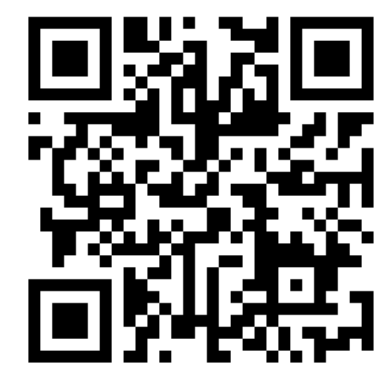

Recibido $11 / 02 / 2021$

\author{
${ }^{1}$ Dr. Kevin Vinicio Loaiza Quirós \\ Investigador independiente, San José, Costa Rica \\ (D) https://orcid.org/0000-0003-1076-156X \\ ${ }^{2}$ Dra. Karen Parada Peña \\ Investigadora independiente, Guanacaste, Costa Rica \\ (1D) https://orcid.org/0000-0001-5405-8548
}

Corregido 18/04/2021
Aceptado

20/04/2021

\section{RESUMEN}

La incontinencia urinaria es un padecimiento con una gran prevalencia, especialmente en la población femenina cuyo riesgo aumenta con la edad. Sin embargo, también se asocia al periodo perinatal, obesidad y deterioro cognitivo. Debido a la carga social y psicológica que conlleva para las pacientes, muchas veces no es documentada y no reciben tratamiento, por lo que se cree que la prevalencia es mayor a la estimada. El tratamiento debe personalizarse, dependiendo del tipo de incontinencia y gravedad de los síntomas.

PALABRAS CLAVE: incontinencia urinaria; incontinencia urinaria de esfuerzo; incontinencia urinaria de urgencia; perimenopausia; menopausia.

\begin{abstract}
Urinary incontinence is a highly prevalent condition, especially in the female population whose risk increases with age, however it is also associated with the perinatal period, obesity and cognitive impairment. Due to the social and psychological burden that it entails for patients, it is often not documented, and they do not receive treatment, so it is believed that the prevalence is higher than estimated. Treatment must be personalized, depending on the type of incontinence and the severity of symptoms.
\end{abstract}

KEYWORDS: urinary incontinence; urinary incontinence stress, urinary incontinence urge perimenopause; menopause.

${ }^{1}$ Médico general, graduado de la Universidad de Costa Rica (UCR). Cód. MED17000. Correo: kevinloaizaquiros@gmail.com

${ }^{2}$ Médica general, graduada de la Universidad de Costa Rica (UCR). Cód. MED16812. Correo: karip 96@hotmail.com 


\section{INTRODUCCIÓN}

Existen distintos tipos de incontinencia urinaria (IU), entre ellos: La incontinencia urinaria de esfuerzo (IUE), incontinencia urinaria de urgencia (IUU) y una mezcla de las dos, la incontinencia urinaria mixta. La micción involuntaria es la característica principal, sin embargo. cada una presenta manifestaciones, abordaje y tratamiento respectivo (1).

Se estima que un número cada vez mayor de mujeres jóvenes y mujeres en edad menopáusica sufrirán incontinencia urinaria. Usualmente ocurre durante los períodos perinatal y perimenopáusico, o bien como resultado de un daño cerebral o un estilo de vida poco saludable (2).

Esta condición aumenta con la edad, llegando a afectar hasta el $77 \%$ de las adultas mayores que residen en hogares de ancianos. A pesar de esta alta prevalencia, solo el $25 \%$ de las mujeres afectadas buscan atención $\mathrm{y}$, de ellas, menos de la mitad reciben tratamiento (3).

El tratamiento para la IU debe personalizarse en función de la frecuencia y gravedad de los síntomas, y de las preferencias y objetivos terapéuticos individuales del paciente (4).

El objetivo de este trabajo es exponer información actualizada sobre la IU, y proporcionar al lector los datos más relevantes de las publicaciones analizadas.

\section{MÉTODO}

La revisión bibliográfica se realizó apoyándose en los buscadores Pubmed y Up to Date; y bases de datos de ScienceDirect, JAMA, Scielo y Nature; empleando frases como "incontinencia urinaria", "incontinencia urinaria de esfuerzo", "incontinencia urinaria de urgencia" y "perimenopausia". Se incluyeron 30 artículos publicados entre el 2016 y el presente año, tanto de generalidades como de distintos enfoques en relación con la incontinencia urinaria, entre ellos fisiopatología y tratamiento de la enfermedad; de los cuales se extrajo la información más relevante para la realización del presente artículo.

\section{PREVALENCIA}

Los estudios de población de numerosos países han informado que la prevalencia de la IU osciló entre aproximadamente el $5 \%$ y el $70 \%$, y la mayoría informaron una prevalencia de cualquier IU en el rango del $25-45 \%$. Esta enorme variación entre estudios se observa tanto dentro de un mismo país como entre países. Las tasas de prevalencia son incluso más altas en las personas de edad avanzada y entre los pacientes de hogares de ancianos (5).

Datos epidemiológicos sugieren una prevalencia global del $17 \%$ en mujeres mayores de 20 años y del $38 \%$ en mujeres mayores de 60 años. Algunos Informes recientes indican que hasta el $37,5 \%$ de las mujeres jóvenes (30-50 años) en un entorno de atención primaria informan incontinencia de esfuerzo (3).

En un estudio de revisión sobre cambios fisiológicos y hormonales durante el proceso de envejecimiento se concluyó que no es razonable considerar los síntomas de tracto urinario inferior (STUI) e incontinencia como algo normal. La incontinencia y los STUI se observan comúnmente junto con la fragilidad en la persona adulta mayor, pero incluso en los pacientes frágiles estos síntomas se pueden controlar, mejorar y contener, debido a que su presencia implica una patología que podría ser susceptible de tratamiento (6).

Varios estudios muestran una prevalencia de IU en el postparto entre 7 y $12 \%$. El inicio de la incontinencia urinaria de esfuerzo durante el primer embarazo o puerperio conlleva un mayor riesgo de síntomas de larga duración, hasta 12 años. Diversos estudios reportan que la incontinencia 
urinaria muestra una tendencia a la reducción en el postparto, pero ocurre de nuevo y en mayor proporción en los siguientes embarazos, por lo que se identifica a la multiparidad como un factor de riesgo para aumento de la frecuencia de esta alteración (7).

La IU también ocurre con frecuencia como consecuencia de una disfunción neurogénica de la vejiga después de una lesión de la médula espinal (LME). A pesar de la abundancia de muchas afecciones de salud secundarias después de una LME, estos pacientes han clasificado los problemas urinarios como el problema de salud más importante como consecuencia de la lesión. En un estudio realizado en Dinamarca con 609 mujeres con lesión espinal, $299 \quad(49 \%)$ experimentaron incontinencia urinaria y esta se asoció a disminución en la calidad de vida en general (8).

\section{FISIOPATOLOGÍA}

\section{Función normal}

La continencia urinaria requiere una interacción compleja entre la actividad del músculo detrusor y la presión de cierre del esfínter uretral controlada por el sistema nervioso. La vejiga está inervada por los sistemas nerviosos parasimpático, simpático y somático que son controlados por los centros corticales superiores y subcorticales. En el almacenamiento de la vejiga, la presión de cierre de la uretra debe exceder la presión de las contracciones del musculo detrusor.

Cualquier aumento de la presión intraabdominal se transmite a la uretra y la vejiga por igual, lo que resulta en continencia. La micción normal se logra cuando la presión uretral desciende y la presión de la vejiga aumenta. Por ende, los problemas con este mecanismo pueden derivar en incontinencia (1).

\section{Incontinencia urinaria por esfuerzo}

En la incontinencia por esfuerzo se produce pérdida de orina asociada a aumento de la presión intraabdominal como por ejemplo: tos, estornudos o esfuerzo físico. Se han descrito dos mecanismos comunes, a menudo superpuestos, para la incontinencia urinaria de esfuerzo: la hipermovilidad uretral resultante de la pérdida de apoyo del cuello de la vejiga y la uretra (de manera que se mueven durante los picos de presión abdominal), y la debilidad del esfínter urinario mismo que puede resultar de un traumatismo, cirugías uroginecológicas repetidas, enfermedad neurológica, envejecimiento 0 enfermedades que conducen a una atrofia muscular sistémica (9).

\section{Incontinencia urinaria por urgencia}

IUU es una fuga involuntaria acompañada o inmediatamente precedida por un repentino y poderoso deseo de orinar que no puede aplazarse o es difícil de aplazar; esta es parte de un complejo de síntomas más amplio conocido como vejiga urinaria hiperactiva (VUH) (1).

Se han propuesto dos posibles vías:

1. Un aumento anormal de las señales aferentes (sensoriales) enviadas desde la vejiga al cerebro. Existen 2 dos teorías sobre este mecanismo: una hipótesis basada en el urotelio y una hipótesis miogénica.

a. Hipótesis basada en el urotelio: establece que los cambios en la función del receptor urotelial, como la regulación ascendente, y en la liberación de neurotransmisores (aumento), así como los cambios en la sensibilidad de la red de células 
suburoteliales pueden conducir a un aumento de la actividad aferente desencadenada por la vejiga $\mathrm{y}$, por tanto, a un aumento de las contracciones involuntarias del músculo detrusor.

\section{b. Hipótesis miogénica: establece} que los cambios en la excitabilidad de las células del músculo liso de la vejiga y de su acoplamiento con otros miocitos o células intersticiales pueden conducir a la generación de contracciones desinhibidas. La denervación parcial del detrusor, debido a inflamación o isquemia, puede cambiar las propiedades de las células del músculo liso, que por lo tanto pueden volverse más excitables y desarrollar un mayor grado de acoplamiento (10).

2. Una capacidad disminuida del cerebro para manejar las señales aferentes provenientes de la vejiga.

Se debe a un manejo anormal de las señales aferentes en el cerebro: el daño a las vías inhibidoras centrales o la sensibilización de las señales aferentes conduce a la pérdida del control de los reflejos miccionales primitivos, lo que desencadena sobreactividad del detrusor. Las lesiones suprapontinas, como las provocadas por un accidente cerebrovascular o la enfermedad de Parkinson, pueden provocar una pérdida de la inhibición voluntaria de la micción. Una lesión de la médula espinal por encima del nivel lumbosacro interrumpe la vía supraespinal descendente por la que se controla voluntariamente la micción. Esto conduce a sobreactividad del detrusor mediada únicamente por vías reflejas espinales (10).

\section{DIAGNÓSTICO Y DETECCIÓN}

Hallazgos de distintos estudios sugieren que existe una pobre correlación general entre el diagnóstico clínico del tipo de incontinencia urinaria y los resultados de las investigaciones urodinámicas, por lo que se sugiere una evaluación clínica cuidadosa y el uso de cuestionarios estandarizados para mejorar la precisión del diagnóstico clínico del tipo de IU en mujeres. El establecimiento del diagnóstico final y la planificación del tratamiento deben basarse en una historia clínica detallada, examen físico, diarios de vejiga e interpretación cuidadosa de los datos urodinámicos (11).

En pacientes perimenopáusicas se debe utilizar la "Menopause Rating Scale", y cuando esta se ve alterada, se debe integrar la aplicación simultánea del cuestionario "International Consultation on Incontinence Questionnaire Short-Form" y "test de Sandvik", con el propósito de establecer el diagnóstico inicial de IU, evaluar la severidad del cuadro, entregar el tratamiento médico o quirúrgico pertinente (12).

Una historia apropiada para un paciente con incontinencia urinaria debe incluir una evaluación de causas reversibles. La mnemotecnia DIAPPERS creado por Resnick proporciona una manera fácil de recordar las causas comunes reversibles de incontinencia, que incluyen (13):

- Delirium

- Infección

- Atrofia vaginal

- Farmacia (pharmaceuticals): antagonistas alfa adrenérgicos, IECA, bloqueadores de canales de calcio, diuréticos, AINES COX 2 selectivos, opioides, relajantes de músculo esquelético, antidepresivos, antipsicóticos, alcohol, antihistamínicos, anticolinérgicos y tiazolidinedionas

- Psicológico: como depresión 
- Exceso de gasto urinario: secundario a consumo excesivo de fluidos, medicación, o diabetes

- Reducción en movilidad

- Constipación (stool impactation)

\section{Factores de riesgo}

Los factores de riesgo importantes de la IU entre las mujeres mayores de la población general incluyen el aumento de la edad, las afecciones médicas preexistentes, el deterioro de la cognición y la disminución de la función física (14).

También el aumento del índice de masa corporal, la diabetes mellitus, la terapia hormonal y el traumatismo o prolapso vaginal parecen estar asociados con el desarrollo de IU en mujeres mayores. En los hombres mayores, la evidencia publicada consistente sugiere que la mala salud general, la limitación en las actividades diarias, el accidente cerebrovascular, la diabetes mellitus y el tratamiento del cáncer de próstata (principalmente cirugía) se asociaron con un mayor riesgo de IU. Además, la hipertrofia prostática benigna es un factor de riesgo común para el desarrollo de IUU en los hombres. Sumado a esto, las condiciones médico-quirúrgicas que padecen, los medicamentos, como diuréticos y psicotrópicos, la polifarmacia y el estado funcional limitado son factores de riesgo de pérdida de continencia en la población adulta mayor frágil (15).

Es importante recordar que los adultos mayores que padecen IU son propensos a infecciones cutáneas, disfunción sexual, pérdida de autoestima, dependencia, depresión, fragilidad, institucionalización, aumento de la carga del cuidador y costo económico. Además, la incontinencia urinaria altera la normalidad de la vida diaria y tiene un impacto en la salud psicosocial que conduce a un deterioro de la calidad de vida (14).

\section{Examen físico}

El médico debe realizar un examen físico de todos los compartimentos de soporte pélvico para descartar factores como divertículos, fístulas y flujo vaginal. También se debe evaluar el prolapso de órganos pélvicos, ya que este puede enmascarar los síntomas de la IU. Se debe efectuar un examen neurológico para determinar si las inervaciones motoras y sensoriales del perineo están intactas. En cuanto a la IUE se debe realizar una prueba de esfuerzo con tos y una evaluación de la movilidad uretral. Una prueba de esfuerzo con tos positiva implica presenciar una fuga de orina simultánea a la tos del paciente. La movilidad uretral se puede medir utilizando la prueba tradicional de "Q-tip", colocando un hisopo de algodón en la uretra y luego midiendo el grado de desplazamiento del cuello uretra-vesical mientras el paciente realiza la maniobra de Valsalva. Un desplazamiento de más de 30 grados con respecto a la horizontal indica hipermovilidad de la unión uretrovesical debido a una disminución del soporte uretral (16).

\section{Exámenes urológicos}

La prueba de orina con tira reactiva, el análisis de orina microscópico y el urocultivo, junto con la medición del volumen residual posmiccional deben realizarse cuando esté indicado para descartar infecciones, hematuria y proteinuria. Se indican evaluaciones adicionales cuando el volumen residual posmiccional es persistentemente superior a $150 \mathrm{ml}$. Los estudios urodinámicos no están indicados en mujeres por lo demás sanas con IUE; estos pueden estar justificadas en mujeres con síntomas complicados de IU, IU refractaria al tratamiento, o historia y resultados dudosos a la exploración física. La evaluación cistoscópica del tracto urinario inferior puede estar indicada en mujeres con IUU 
refractaria al tratamiento, pérdida continua de orina que haga sospechar de lesiones o fístulas genitourinarias iatrogénicas, goteo persistente posmiccional y hematuria (17).

\section{MANEJO}

\section{Intervenciones no quirúrgicas}

El objetivo del estudio "Group Learning Achieves Decreased Incidents of Lower Urinary Symptoms" (GLADIOLUS) fue evaluar la efectividad, el costo y la rentabilidad de una sola clase grupal de salud de la vejiga de 2 horas para ofrecer un programa de tratamiento conductual basado en evidencia complementado con materiales para guiar la práctica en el hogar para mujeres mayores con urgencia, estrés o IU mixta. Este estudio concluyo que este enfoque de terapia conductual grupal es seguro y efectivo para reducir la frecuencia, la gravedad y las molestias de la IU y mejorar la calidad de vida de las mujeres mayores en la comunidad. Con su bajo costo y facilidad de administración, es un primer enfoque prometedor para mejorar el acceso al tratamiento conductual no invasivo para IU (18).

Como primera intervención, se encuentran las recomendaciones de terapia conductual: El entrenamiento de la vejiga consiste en la micción guiada o programada cada 1 hora, aumentando media hora cada semana hasta un objetivo de dos y media a tres horas entre micciones. Los ejercicios del músculo pélvico (EMPP) consiste en al menos 15 contracciones de los músculos del suelo pélvico tres veces al día, cada una de las cuales dura lo más cerca posible de 10 segundos para el paciente. Esto puede ser con o sin biofeedback, a discreción del médico. Entre las instrucciones para manejar la IUU se encuentran: No apresurarse a ir al baño por la sensación de urgencia, sino sentarse e intentar relajarse y realizar 3 a 4 contracciones rápidas de los músculos del piso pélvico, una vez que pase la sensación, continuar yendo al baño a un ritmo normal. Mientras que para IUE: Contraer los músculos del piso pélvico antes y durante la realización de la actividad asociada con la incontinencia (toser y estornudar) (19).

Una revisión de Cochrane que incluyó 31 ensayos con 1817 mujeres de 14 países tenía como objetivo evaluar los efectos del EMPP para las mujeres con IU en comparación con ningún tratamiento, placebo o tratamientos simulados $u$ otros tratamientos de control inactivos. En cuanto a la curación sintomática de la IU al final del tratamiento en comparación con ningún tratamiento o con tratamientos de control inactivo, las mujeres con IUE que estaban en los grupos de EMPP tenían ocho veces más probabilidades de informar la curación (56\% versus $6 \%$ ); para las mujeres con cualquier tipo de IU, los grupos de EMPP tenían cinco veces más probabilidades de informar la curación (35\% versus $6 \%$ ). Según los datos disponibles, podemos estar seguros de que el EMPP puede curar o mejorar los síntomas de la IUE y todos los demás tipos de IU. Además, puede reducir el número de episodios de fugas, y los síntomas en los cuestionarios de síntomas específicos de la IU, así como mejorar la calidad de vida de las pacientes (20).

En otra revisión por Cochrane sobre la efectividad de EMPP en la prevención o el tratamiento de la incontinencia urinaria y fecal en mujeres embarazadas o posparto, se documentó que las mujeres embarazadas continentes que realizan EMPP prenatal pueden haber tenido un riesgo menor de informar incontinencia urinaria al final del embarazo (62\% menos). De manera similar, el EMPP prenatal redujo el riesgo de incontinencia urinaria (29\% menos) en el período posnatal medio (más de tres a seis meses después del parto). No hubo información suficiente disponible para el período posnatal tardío (más de seis a 12 
meses) para determinar los efectos en este momento (21).

Además de las intervenciones clínicas o cuando el tratamiento no ha permitido recuperar la continencia, existen distintas ayudas que pueden permitir a las personas continuar con sus actividades diarias y mantener una buena calidad de vida. Las compresas para incontinencia evitan que la orina contamine la ropa, los muebles y la ropa de cama, lo que permite al individuo mantener los síntomas en privado. La selección de la almohadilla depende de la cantidad de orina perdida y de cuándo, durante el día y la noche, se experimenta. EI paciente debe usar la almohadilla más pequeña que sea apropiada para la cantidad de orina perdida. Otra opción es la de la gama de compresas y pantalones lavables, especialmente adecuada cuando los pacientes experimentan reacciones cutáneas a las compresas desechables (22). Los conos vaginales y pesarios también pueden ser utilizados como primera línea de tratamiento en la IUE, o en caso de que los cambios en el comportamiento y los EMPP no hayan logrado el efecto esperado (23).

\section{Intervenciones medicamentosas}

En pacientes postmenopausicas en comparación con el placebo, el estrógeno tópico resultó en una mejora del $50 \%$ en los episodios de IUE. De manera similar, para los pacientes con IUU después de 1 año de terapia con estrógenos, la incontinencia mejoró en un $50 \%$.

Para las mujeres con IUU, una revisión Cochrane de 32 ensayos controlados aleatorios demostró que los antimuscarínicos reducen la frecuencia y la urgencia asociadas con la VUI y son eficaces en hasta el $75 \%$ de los pacientes. Se debe advertir a los pacientes que pueden tardar hasta 4 semanas en actuar, se pueden tomar a largo plazo y si un medicamento falla, otro medicamento de la misma clase podría tener éxito. Los efectos secundarios predominantes son boca seca, estreñimiento, visión borrosa, fatiga y deterioro cognitivo, y están relacionados con la dosis.

Mirabegrón es el primer agonista de los adrenorreceptores beta 3 autorizado para su uso en el tratamiento de la VUH. Este conduce a la relajación del músculo liso a través de una vía de AMP cíclico y una reducción del calcio citoplasmático. Se recomienda para pacientes con IUU en los que los antimuscarínicos no funcionaron debido a la falta de eficacia o por efectos secundarios intolerables.

La duloxetina es un inhibidor de la recaptación de serotonina y noradrenalina que actúa en la médula espinal sacra en el núcleo de Onuf para aumentar la actividad del nervio pudendo y aumentar el tono de los músculos uretrales. Su uso en la IUE fue respaldado por una amplia revisión en 2007 que mostró una reducción en el número de episodios de fuga, un aumento en el intervalo miccional y un aumento en la calidad de vida. Sin embargo, a pesar de una eficacia del 20 al $40 \%$, tiene poca tolerancia donde un $30 \%$ de los pacientes sufrieron efectos secundarios, lo que llevó a un $10 \%$ a suspender la medicación.

\section{Intervenciones invasivas}

Para IUE la primeria línea de tratamiento quirúrgico en la eslinga uretral ya sea retropúbica o transobturadora, también puede utilizarse en IU mixta. De forma alternativa y ante recurrencias, se pueden utilizar inyecciones ocluyentes periuretrales o un esfínter uretral artificial (23).

En casos de IUU se puede utilizar inyección de toxina botulínica como tratamiento de segunda línea, después de la terapia conductual e intervenciones medicamentosas. La estimulación percutánea de nervio tibial también es utilizada como tratamiento para IUU 
refractaria. Mientras que la cistoplastía de aumento es una opción en caso de las terapias anteriores hayan fallado (23).

En cuanto a neuromodulación sacra, de acuerdo con una revisión de datos realizada en Nueva York se encontró un número alarmante de reintervenciones, principalmente debido al mal funcionamiento del dispositivo para todas las indicaciones. Uno de cada 3 pacientes tuvo alguna forma de reintervención, ya sea revisión o extracción, dentro de los 3 a 5 años de la colocación inicial del dispositivo. Concluyen que se necesita con urgencia un registro de dispositivos en este entorno para asesorar a las partes interesadas y ayudar a futuras innovaciones (25).

De acuerdo con un estudio realizado en Estados Unidos, cuyo objetivo era determinar si la combinación de la terapia muscular conductual y del piso pélvico con la eslinga mediouretral es más efectiva que la eslinga sola para mejorar los síntomas mixtos de incontinencia urinaria se reportó que hubo una pequeña diferencia estadísticamente significativa entre grupos que no alcanzó el umbral de importancia clínica establecido por el estudio (26).

La incontinencia urinaria se desarrollará después de la reparación del prolapso en aproximadamente una cuarta parte de las pacientes con prolapso avanzado de órganos pélvicos. Muchas mujeres con prolapso avanzado de órganos pélvicos que optan por someterse a un tratamiento quirúrgico también optan por someterse a una cirugía de continencia para prevenir la incontinencia urinaria de nueva aparición. EI cabestrillo/eslinga mediouretral, la cistouretropexia de Burch y el cabestrillo para el cuello de la vejiga son opciones de tratamiento muy eficaces para la incontinencia urinaria de esfuerzo en estas situaciones.

Además, múltiples ensayos demuestran que más del $80 \%$ de estas mujeres que se quejan de incontinencia preoperatoria, se curan o tienen una mejoría significativa en sus síntomas hasta 5 años después de la colocación de cabestrillos mediouretrales (27).

\section{CALIDAD DE VIDA}

La presencia de incontinencia urinaria se asocia al estigma, miedo, y vergüenza relacionados con la condición clínica, con repercusión en la autoestima y alteración de la vida personal, social y sexual (28).

Hay literatura que evidencia que del 70 al 80 $\%$ de las mujeres climatéricas sufre de alguna sintomatología urogenital durante la perimenopausia. Se han descrito tanto efectos en la esfera sexual y genitourinaria como son la incontinencia urinaria coital, la enuresis nocturna y prurito genital, así como efectos psicoemocionales entre ellos vergüenza, miedo, reducción del deseo sexual, angustia, ansiedad y depresión (12). Una revisión sistemática de 18 estudios determinó que la IU tiene un impacto negativo en la función sexual.

Es probable que esta injerencia, directa o indirectamente, contribuya de manera decisiva que las mujeres eviten tener relaciones sexuales. Los estudios indican que hay una mayor prevalencia de abstinencia sexual, menos deseo sexual, juegos previos, armonía con la pareja, comodidad y satisfacción sexual en esas mujeres (29).

Según la OMS, del 28 al $35 \%$ de las personas mayores de 65 años presentan al menos una caída cada año y la prevalencia de caídas aumenta con la edad. En un estudio coreano que incluyó a un total de 6.134 mujeres de entre 65 y 106 años, con una edad media de 74,8 años, se documentó un posible aumento del riesgo de caídas y caídas recurrentes en personas mayores con IU en comparación con quienes no la padecen, especialmente en personas con deterioro cognitivo y funcional (30). 


\section{CONCLUSIONES}

A pesar de que los estudios reportan una alta prevalencia de este padecimiento, se cree que es subdiagnosticado. Es importante que el personal de salud indague de forma activa sobre los síntomas en las pacientes de riesgo, y que se apoye en las distintas escalas y cuestionarios, así como en los estudios urodinámicos para establecer el diagnostico.

Se debe hacer énfasis en adoptar estrategias conductuales como reducir la ingesta de cafeína, beber pequeñas cantidades de líquido con frecuencia en lugar de grandes cantidades de líquido a la vez, micción programada y ejercicios de los músculos del piso pélvico. Además de lo anterior, para la IUU existen medicamentos con el fin de mejorar la sintomatología, mientras que para la IUE hay dispositivos vaginales, incluidos pesarios o insertos de venta libre. También se puede considerar la cirugía u otros procedimientos si esta persiste a pesar de las terapias más conservadoras.

El abordaje y tratamiento oportuno, conlleva una mejoría en la calidad de vida de las pacientes, con resultados tanto en el aspecto biológico como en el psicológico y social, de allí la importancia de una intervención adecuada.

\section{Los autores declaran no tener conflicto de interés.}

\section{REFERENCIAS}

1. Bardsley A. An overview of urinary incontinence. British Journal of Nursing. 2016;25(18):S14-S21. https://doi.org/10.12968/bjon.2016.25.18.S14

2. Kopańska M, Torices S, Czech J, Koziara W, Toborek M, et al. Urinary incontinence in women: biofeedback as an innovative treatment method. Therapeutic Advances in Urology. 2020;12. https://doi.org/10.1177/1756287220934359

3. Lukacz ES, Santiago-Lastra Y, Albo ME, Brubaker L. Urinary Incontinence in Women: A Review. JAMA. 2017;318(16):1592-1604. https://doi.org/10.1001/jama.2017.12137
4. Muth CC. Urinary Incontinence in Women. JAMA. 2017;318(16):1622.

https://doi.org/10.1001/jama.2017.15571

5. Milsom I, Gyhagen M. The prevalence of urinary incontinence. Climacteric. 2019;22(3):217-222. https://doi.org/10.1080/13697137.2018.1543263

6. Gibson W, Wagg A. Incontinence in the elderly, 'normal' ageing, or unaddressed pathology? Nature Reviews Urology. 2017 Jul;14(7):440-448. https://doi.org/10.1038/nrurol.2017.53

7. Díaz F, Fuentes M, Rivadeneira A, Acuña L. Prevalencia de incontinencia urinaria en el posparto. Revista Cubana de Obstetricia y Ginecología. 2017;43(2):1-11. Disponible en: http://www.revginecobstetricia.sld.cu/index.php/gi n/article/view/209

8. Elmelund $\mathrm{M}$, Klarskov $\mathrm{N}$, Biering-Sørensen $\mathrm{F}$. Prevalence of urinary incontinence in women with spinal cord injury. Spinal Cord. 2018 Dec;56(12):1124-1133. https://doi.org/10.1038/s41393-018-0157-0

9. Aoki Y, Brown H, Brubaker L, Cornu JN, Daly JO, et al. Urinary incontinence in women. Nature Reviews Disease Primers. 2017;3(17042). https://doi.org/10.1038/nrdp.2017.42

10. Wyndaele M, Hashim H. Pathophysiology of urinary incontinence. Surgery (Oxford). 2017;35(6):287-292, https://doi.org/10.1016/j.mpsur.2017.03.002

11. Palaiologos K, Annappa M, Grigoriadis G. Correlation Between Urodynamic and Clinical Diagnoses in Classifying the Type of Urinary Incontinence in Women. Cureus. 2019;11(10):e6016. https://doi.org/10.7759/cureus.6016

12. Silva MA, Gallardo M, López C, Santander C, Torres J. Efectos de la incontinencia urinaria en la calidad de vida de la mujer climatérica. Revista Cubana de Obstetricia y Ginecología. 2018;44(1): 1-14. Disponible en: http://revginecobstetricia.sld.cu/index.php/gin/arti cle/view/307

13. Irwin GM. Urinary Incontinence. Primary Care: Clinics in Office Practice. 2019;46(2):233-242. https://doi.org/10.1016/j.pop.2019.02.004

14. Murukesu RR, Singh DKA, Shahar S. Urinary incontinence among urban and rural community dwelling older women: prevalence, risk factors and quality of life. BMC Public Health. 2019;19(Suppl 4):529. https://doi.org/10.1186/s12889-019-6870$\underline{6}$

15. Aharony L, De Cock J, Nuotio MS, Pedone C, Rifel $\mathrm{J}$, et al. Consensus document on the detection and diagnosis of urinary incontinence in older people. European Geriatric Medicine. 2017;8(3):202-209. https://doi.org/10.1016/i.eurger.2017.03.012

16. Goforth J, Langaker M. Urinary Incontinence in Women. North Carolina Medical Journal. 
2016;77(6):423-425.

https://doi.org/10.18043/ncm.77.6.423

17. Dufour S, Wu M. No. 397 - Conservative Care of Urinary Incontinence in Women. Journal of Obstetrics and Gynaecology Canada. 2020;42(4):510-522. https://doi.org/10.1016/j.jogc.2019.04.009

18. Diokno AC, Newman DK, Low LK, Griebling TL, Maddens ME, et al. Effect of Group-Administered Behavioral Treatment on Urinary Incontinence in Older Women: A Randomized Clinical Trial. JAMA Internal Medicine. 2018;178(10):1333-1341. https://doi.org/10.1001/jamainternmed.2018.3766

19. Kilpatrick KA, Paton $P$, Subbarayan S, Stewart C, Abraha I, et al. Non-pharmacological, non-surgical interventions for urinary incontinence in older persons: A systematic review of systematic reviews. The SENATOR project ONTOP series. Maturitas. 2020;133:42-48. https://doi.org/10.1016/j.maturitas.2019.12.010

20. Dumoulin C, Cacciari LP, Hay-Smith EJC. Pelvic floor muscle training versus no treatment, or inactive control treatments, for urinary incontinence in women. Cochrane Database of Systematic Reviews 2018;(10). Art. No.: CD005654. https://doi.org/10.1002/14651858.CD005654.pub $\underline{4}$

21. Woodley SJ, Boyle R, Cody JD, Mørkved S, Hay-Smith EJC. Pelvic floor muscle training for prevention and treatment of urinary and faecal incontinence in antenatal and postnatal women. Cochrane Database of Systematic Reviews. 2017;(12). Art. No.: CD007471. https://doi.org/10.1002/14651858.CD007471.pub $\underline{3}$

22. Wilson M. Urinary incontinence: considering the physical and psychological implications. British Journal of Community Nursing. 2016;21(5):222-4. https://doi.org/10.12968/bjcn.2016.21.5.222

23. Favre-Inhofer A, Dewaele $P$, Millet $P$, Deffieux $X$. Systematic review of guidelines for urinary incontinence in women. Journal of Gynecology Obstetrics and Human Reproduction. 2020:101842. https://doi.org/10.1016/j.jogoh.2020.101842

24. Yang B, Foley S. Overview on the management of adult urinary incontinence. Surgery (Oxford). 2020;38(4):204-211, https://doi.org/10.1016/j.mpsur.2020.01.016

25. Chughtai B, Thomas D, Sun T, Sedrakyan A. Failures of Sacral Neuromodulation for Incontinence. JAMA Surgery. 2018;153(5):493$494 \mathrm{https}: / /$ doi.org/10.1001/jamasurg.2017.6093

26. Sung VW, Borello-France D, Newman DK, Richter HE, Lukacz ES, et al. Effect of Behavioral and Pelvic Floor Muscle Therapy Combined With Surgery vs Surgery Alone on Incontinence
Symptoms Among Women With Mixed Urinary Incontinence: The ESTEEM Randomized Clinical Trial. JAMA. 2019;322(11):1066-1076. https://doi.org/10.1001/jama.2019.12467

27. Jelovsek JE. Predicting urinary incontinence after surgery for pelvic organ prolapse. Current Opinion Obstetrics Gynecology. 2016 Oct;28(5):399-406 https://doi.org/10.1097/GCO.0000000000000308

28. Mota RL. Female urinary incontinence and sexuality. Int Braz J Urol. 2017;43(1):20-28. https://doi.org/10.1590/s16775538.ibju.2016.0102

29. Pinheiro LR, Fernandez D, Ribeiro IP, Lira do Nascimento S, Morais AV, et al. The Impact of Urinary Incontinence on Sexual Function: A Systematic Review. Sexual Medicine Reviews. 2020;8(3):393-402. https://doi.org/10.1016/j.sxmr.2019.06.009

30. Moon S, Chung HS, Yu JM, Na HR, Kim SJ, et al. "Impact of Urinary Incontinence On Falls in the Older Population: 2017 National Survey of Older Koreans." Archives of Gerontology and Geriatrics. 2020;90:104158. https://doi.org/10.1016/j.archger.2020.104158 